\title{
Optical vortices and the flow of their angular momentum in a multimode fiber
}

\author{
A. N. Alexeyev, T. A. Fadeyeva, A. V. Volyar \\ Physical Department, Simferopol State University, Yaltinskaya 4, 333036 Simferopol, Ukraine
}

\author{
M. S. Soskin \\ Institute of Physics, NAS Ukraine, 46 prospekt Nauki, Kyiv, 252028, Ukraine
}

\begin{abstract}
The problem of propagation of optical vortices in multimode fibers is considered. The structural changes experienced by the wave and ray surfaces in their transformation from the free space to a fiber medium are determined. The continuity equation is obtained for the flow of the vortex angular momentum in an unhomogeneous medium.
\end{abstract}

Keywords: optical vortex, multimode fiber, angular momentum, continuity equation.

Paper received 23.06.98; revised manuscript received 14.08.98; accepted for publication 28.10.98.

\section{Introduction}

Defects of the radiation wavefront in a multimode fiber are usually associated with a distortion of the field structure. Thus, it can be concluded that such effects hinder the use of multimode fibers in optical communication lines and sensors. However, a thorough study of the nature of wave deficiency makes us take a new look on the problem of data transmission through a multimode optical fiber, where waves with wavefront defects play the main role.

Defects of a scalar wave field structure were explicitly studied in [1] by J. Nay and M. Berry. They classify such defects by dividing them into purely screw, purely edge and mixed screw-edge dislocations of the wavefront. This classification is based on the fact that the real and imaginary parts of the field strength should be simultaneously equal to zero

$\operatorname{Re}[e(x, y, z)]=0, \quad \operatorname{Im}[e(x, y, z)]=0$.

The problem of light beams in the free space deals mainly with the issues of generation of wavefront dislocations inside laser resonators [2], on phase optical holograms [3, 4] or on an astigmatic mode converter [5]. Sometimes a light field with a purely screw dislocation is called an optical vortex [2].

Wavefront dislocations in a multimode fiber field were first described in [6], and a correlation was found to exist between the average number of dislocations and the number of fiber eigenmodes. Purely screw dislocations are experimentally observed in the form of «forks» in the interference pattern. Changes of external conditions result in a movement, birth, and death of random dislocations in the optical fiber $[7,8]$.
Studies of the properties of single optical vortices in optical fibers have been started quite recently due to development of low mode fiber applications, techniques of selective excitation [9] and radiation field isolation from a fiber $[10,11]$. There is certain difference between optical vortices in a fiber and in the free space. First of all, it applies to the relation between the light wave polarization $\sigma_{z}$ and its topological charge $l$. The helicity $\sigma_{z}$ characterizes the direction of vector rotation of the electric (magnetic) field of a light beam $\left(\sigma_{z}=+1\right.$ is right and $\sigma_{z}=-1$ is left circular polarization).

For a paraxial Gaussian beam in the free space, it is possible to change the values and signs of the topological charge $l$ and helicity $\sigma_{z}$ independently [11].

Amazing properties of optical vortices in the free space were presented in $[12,13]$. It was shown that an optical vortex transmits the angular momentum which can be calculated as

$\mathbf{M}=\frac{1}{c^{2}} \int_{S} \mathbf{r} \times \mathbf{P} d S$

where $\mathbf{r}$ is the radius-vector, $\mathbf{P}$ is the Pointing vector, $\mathrm{S}$ is the area of beam's cross section. When passing through the mode converter, the angular momentum $\mathbf{M}$ is able to change its value and sign [14].

Moreover, the remarkable experiments recently reported in [15] have shown that an optical vortex can trap and screw microscopic particles. Change of the polarization $\sigma_{z}$ of a Gaussian beam may change the state of these particles [15]. Contrary to optical vortices in the free space, guided vortices in an optical fiber are rigidly defined by the pair of numbers: the topological charge $l$ and helicity $\sigma$. Values of $l$ and 


\section{K. N. Alexeyev et al.: Optical vortices and angular momentum...}

$\sigma_{z}$ cannot be changed independently from one another. In addition, the requirement for an optical vortex to be stable is determined by the selection rule $l+\sigma_{z} \neq 0$ [18].

Thus, a question arises about the possibility to use fields in optical fibers for practical purposes. In an optical fiber, a set of spatially distributed optical vortices may exist simultaneously. Furthemore, by means of an optical fiber a light vortex can be placed in locations where it would be impossible to use conventional optical devices.

The objective of this paper is to study the properties of optical vortices and their angular momenta in the field of a multimode optical fiber.

In the second section we, consider eigenfields of an axially symmetric low-mode fiber presented in a circularly polarized basis. The angular momentum flow, the continuity equation for the angular momentum flow, and correlation with that in the free space are analyzed in the third, fourth and fifth sections of this paper.

\section{Guided vortices in an optical fiber}

Consider the peculiarities of the propagation of circularly polarized waves in a locally isotropic axially symmetrical medium of a multimode optical fiber with the refractive index:

$n^{2}(R)=n_{c o}^{2}(1-2 \Delta f(R))$,

where $n_{c o}$ is the refractive index along the fiber axis for $R=$ $=0, R=\rho / \rho_{0}, \rho$ is the radial coordinate, $\rho_{0}$ is the core radius, $\Delta=\left(\frac{n_{c o}^{2}-n_{c l}^{2}}{2 n_{c o}^{2}}\right), n_{c l}$ is the refractive index of clad, $f(R)$ is the function of the refractive index profile.

The stationary vector wave equation for the electric field strength in an inhomogeneous medium can be written in following form:

$$
\left\{\nabla_{t}^{2}+k^{2} n^{2}-\beta^{2}\right\} \mathbf{e}_{\mathbf{t}}=-\nabla_{t}\left\{\mathbf{e}_{\mathbf{t}} \cdot \nabla_{t} \ln n^{2}\right\},
$$

where $k$ is the wave number in vacuum, $\beta$ is the propagation constant.

For weak guiding fibers with a low energy loss $n$ is a real value and $n_{c o} \approx n_{c l}$, then the profile parameter $\Delta$ can be written as

$$
\Delta \approx\left(n_{c o}-n\right) / n_{c l} .
$$

In this case, we can neglect the term in the right side of equation (4) [16] and rewrite the wave equation in the form

$$
\left\{\nabla_{t}^{2}+k^{2} n^{2}-\beta^{2}\right\} \mathbf{e}_{\mathbf{t}}=0,
$$

where $\widetilde{\beta}$ is the propagation constant in scalar approximation, $\tilde{\mathbf{e}}_{t}$ is the electric field in this approximation,

$$
\nabla_{t}^{2} \equiv \frac{\partial^{2}}{\partial x^{2}}+\frac{\partial^{2}}{\partial y^{2}} \text {. }
$$

If the field distortion $\mathbf{e}$ in a weak guiding fiber in the limit $\Delta \rightarrow 0$ in equation (4) is assumed to be small, then $\mathbf{e} \approx \widetilde{e}$, and the propagation constant $\beta=\widehat{\beta}+\delta \beta$, where $\delta \beta$ is the polarization correction. From equations (4) and (6) it is possible to obtain

$$
\delta \beta=\frac{(2 \Delta)^{3 / 2}}{2 \rho_{0} V} \int_{\theta_{\infty}}\left(\rho_{0} \nabla_{t} \cdot \mathbf{e}_{\mathbf{t}}\right)\left\{\mathbf{e}_{\mathbf{t}} \cdot \rho_{0} \nabla_{t} f\right\} d \theta / \int_{\theta_{\infty}} \cdot \mathbf{e}_{t}^{2} d \theta,
$$

where $\theta_{\infty}$ is the cross section area.

The solution of equation (6) can be obtained in the form:

$\mathbf{e}=\hat{\mathbf{e}}^{ \pm} \exp \{ \pm i l \varphi\} F_{l}(R) \exp \{i \widehat{\beta} z\}$

where $\hat{\mathbf{e}}^{ \pm}$is the unit vector of the right $(+)$or left $(-)$circular polarization, $\varphi$ is the azimuth coordinate, $l$ is the azimuth index $(l=0,1,2, \ldots)$. The radial function $F_{l}(R)$ is obtained from equation [16]

$\left\{\frac{d^{2}}{d R^{2}}+\frac{1}{R} \frac{d}{d R}-\frac{l^{2}}{R^{2}}+V^{2}-V^{2} f\right\} F(R)=0$,

where $\sigma$ is the waveguide mode parameter in the fiber core, determined from the boundary conditions.

Taking into account in (8) particular solutions of equations (7), its can be shown that there are three groups of eigenmodes [17]:

1) Circularly polarized homogeneous optical $\mathrm{CV}$ vortices for $l=1,2,3 \ldots$ ("CV" is for circular vortex [9])

$$
\left[\begin{array}{l}
\mathbf{e}_{\mathbf{t}}=\hat{\mathbf{e}}^{ \pm} F_{l}(R) \exp \{ \pm i l \varphi\} \\
e_{z}=i \frac{\sqrt{2 \Delta}}{V} G_{l}^{-}(R) \exp \{ \pm i(l+1) \varphi\} \\
\mathbf{h}_{t}=-i \hat{\mathbf{e}}^{ \pm} n_{c o} \sqrt{\varepsilon_{0} / \mu_{0}} F_{l}(R) \exp \{ \pm i l \varphi\} \\
h_{z}=n_{c o} \sqrt{\varepsilon_{0} / \mu_{0} \frac{\sqrt{2 \Delta}}{V}} G_{l}^{-}(R) \exp \{ \pm i(l+1) \varphi\}
\end{array}\right] \times \exp \left\{i \beta_{1} z\right\}
$$

2) Circularly polarized unhomogeneous optical CV vortices $(l>1)$

$$
\left[\begin{array}{l}
\mathbf{e}_{\mathbf{t}}=\hat{\mathbf{e}}^{\mp} F_{l}(R) \exp \{ \pm i l \varphi\} \\
e_{z}=i \frac{\sqrt{2 \Delta}}{V} G_{l}^{+}(R) \exp \{ \pm i(l-1) \varphi\} \\
\mathbf{h}_{t}=-i \hat{\mathbf{e}}^{\mp} n_{c o} \sqrt{\varepsilon_{0} / \mu_{0}} F_{l}(R) \exp \{ \pm i l \varphi\} \\
h_{z}=n_{c o} \sqrt{\varepsilon_{0} / \mu_{0} \frac{\sqrt{2 \Delta}}{V}} G_{l}^{+}(R) \exp \{ \pm i(l-1) \varphi\}
\end{array}\right] \times \exp \left\{i \beta_{2} z\right\}
$$

3) Linearly polarized azimuth-symmetrical fields $(l=1)$

$$
\mathrm{TM}_{0 \mathrm{~m}}:\left(\begin{array}{l}
\mathbf{e}_{\mathbf{t}}=(\hat{\mathbf{x}} \cos \varphi+\hat{\mathbf{y}} \sin \varphi) F_{1}(R) \\
e_{z}=i \frac{\sqrt{2 \Delta}}{V} G_{1}^{+}(R) \\
h_{t}=-n_{c o} \sqrt{\varepsilon_{0} / \mu_{0}}(\hat{\mathbf{x}} \sin \varphi-\hat{\mathbf{y}} \cos \varphi) F_{1}(R) \\
h_{z}=0
\end{array}\right) \times \exp \left(i \beta_{3} z\right)
$$




\section{K. N. Alexeyev et al.: Optical vortices and angular momentum...}

$\mathrm{TE}_{0}:\left(\begin{array}{l}\mathbf{e}_{\mathbf{t}}=(\hat{\mathbf{x}} \sin \varphi-\hat{\mathbf{y}} \cos \varphi) F_{1}(R) \\ e_{z}=0 \\ h_{t}=n_{c o} \sqrt{\varepsilon_{0} / \mu_{0}}(\hat{\mathbf{x}} \cos \varphi+\hat{\mathbf{y}} \sin \varphi) F_{1}(R) \\ h_{z}=i n_{c o} \sqrt{\varepsilon_{0} / \mu_{0}} \frac{\sqrt{2 \Delta}}{V} G_{1}^{+}(R)\end{array}\right) \times \exp \left(i \beta_{4} z\right)$

where $V=\frac{2 \pi}{\lambda} \rho n_{c o} \sqrt{2 \Delta}$ is the waveguide fiber parameter, $G_{1}^{\mathrm{\kappa}}(R)=\frac{d F_{1}(R)}{d R}+\kappa \frac{F_{1}(R)}{R}, \beta_{i}$ are the propagation constants of eigenmodes obtained from the dispersion equation [16], $\kappa= \pm 1$ is the spin-orbital interaction index. In scalar approximation, $\delta \beta_{i}=0$, and $\beta_{i}$ of all eigen modes for a given index $l$ are degenerated with respect to the propagation constant $\beta$.

The expressions (10)-(13) form an orthogonal set of functions, describing fields of guided modes on the lengths of the so-called stated regime, when the fields of radiated and tunnelling modes are negligibly small [16].

Expression (10) for $l=0$ describes the field of the fundamental circularly polarized $\mathrm{HE}_{11}$ mode [1]:

$$
\left(\begin{array}{l}
\mathbf{e}_{\mathbf{t}}=\hat{\mathbf{e}}^{ \pm} F_{0}(R) \\
e_{z}=i \frac{\sqrt{2 \Delta}}{V} G_{0}(R) \exp \{ \pm i \varphi\} \\
\mathbf{h}_{t}=-i \hat{\mathbf{e}}^{ \pm} n_{c o} \sqrt{\varepsilon_{0} / \mu_{0}} F_{0}(R) \\
h_{z}=n_{c o} \sqrt{\varepsilon_{0} / \mu_{0} \frac{\sqrt{2 \Delta}}{V}} G_{0}(R) \exp \{ \pm i \varphi\}
\end{array}\right] \times \exp \left\{i \beta_{0} z\right\}
$$

Let us analyse the wavefront structure of fiber eigenmodes and compare it with the wavefront structure in a void. In the expression (10), the transversal electric field component $\mathbf{e}_{\mathbf{t}}$ of a homogeneous $\mathrm{CV}$ vortex has a circular polarization and is characterised by the profile function $F_{l}(R)$. For axially symmetrical fibers, the value $F_{l}(R)$ turns into zero at the fiber axis $(R=0)$, and far away from the axis $(R \rightarrow \propto)$. For instance, for a fiber with a parabolic index, the value of $F_{l}(R)$ is $F_{l}(R)=R^{|l|} L_{m}{ }^{(l)}(R) \exp \left(-1 / 2 V R^{2}\right)$ (V is the waveguide parameter). It is shown in Fig. 1. Therefore, in a homogeneous $\mathrm{CV}$ vortex field, the factor $R^{|l|} \exp (i l \varphi)$ may be selected. However, the envelope of the optical vortex wavefront in a void has a parabolic form (Fig. 2, a), while the envelope of the transversal component of a circular vortex has a quasiplanar form (Fig. 2, b). This points to the fact that the transversal CV vortex field (10) has a purely screw dislocation with a topological charge $l$.

The longitudinal $e_{z}$ component of a CV vortex turns into zero at the fiber axis (Fig.1) $G_{l}^{-}(R=0)=0$. However, its topological charge equals to $l+1$.

The transversal component of the unhomogeneous CV vortex $(|l|>1)$ in expression (11) is also circularly polarized and has a purely screw wavefront dislocation with index $l$. The longitudinal components of this vortex turns into zero at the axis $\mathrm{z}\left(G_{l}^{+}(R=0)=0, l \neq 0,1\right)$, but its topological charge equals to $l-1$.
The transversal components of the $\mathrm{TM}_{0 \mathrm{~m}}$ and $\mathrm{TE}_{0 \mathrm{~m}}$ modess $(l=1)$ have azimuth-symmetrical distribution of the electric $\mathbf{e}_{\mathbf{t}}$ and magnetic $\mathbf{h}_{\mathbf{t}}$ fields (see (12), (13)).

At the fiber axis, these fields turn into zero. In accordance with the polarization singularities [19] we conclude that these fields have purely screw declinations of polarization.

It should be noted that the electric (magnetic) field of a Gaussian beam in a void has also a longitudinal $e_{z}$ (or $h_{z}$ ) component. For instance, the $e_{z}$ component of the electric field may be represented in the following form:

$e_{z}=\frac{\sigma}{\omega \varepsilon_{0}}\left(\frac{\partial e_{x}}{\partial \rho}+i \sigma \frac{\partial e_{x}}{\partial \varphi}\right) \exp \{i \sigma \varphi\}$

(paraxial approximation).

Excitation of optical fibers is performed in such a way that the entrance of a fiber is placed in the region of the Gaussian beam necking, and the fiber axis and the beam axis coincide. The curvature radius of the wavefront beam in the necking is $R \rightarrow \propto$, and the transversal component of the $\mathrm{CV}$ field also has the curvature radius $R \rightarrow \propto$.

The identical structure of the optical vortices in a void and the guided CV vortices in a fiber permit the laser fields and the eigen fiber fields to be aligned with a high precision (fig. 2).

The transversal fields of the $\mathrm{HE}_{11}$ mode $\mathrm{e}_{\mathrm{t}}$ and $\mathrm{h}_{\mathrm{t}}$ do not have phase singularities. If the longitudinal $e_{z}$ and $h$ field components are assumed to be a transversal wave propagating along the azimuth coordinate $\varphi$, then this field has a purely screw dislocation of the wave front with the topological charge $l=1$ at $R=0$. It is evident from the fact that all smooth values of the refractive index profiles $n(R)$ for the function $G_{0}(R=0)$ are equal to zero on the fiber axis.

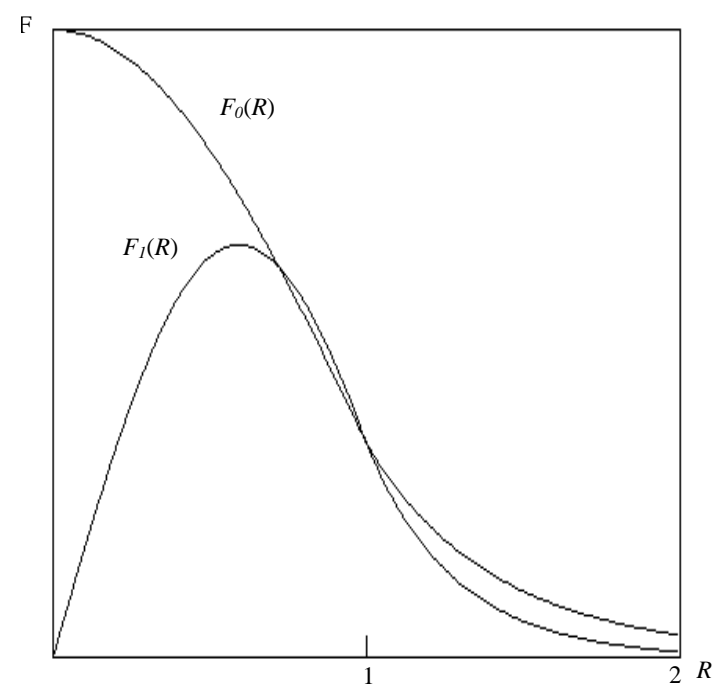

Fig. 1. The field strength profile function $F_{l}(R)$. 


\section{K. N. Alexeyev et al.: Optical vortices and angular momentum...}

a

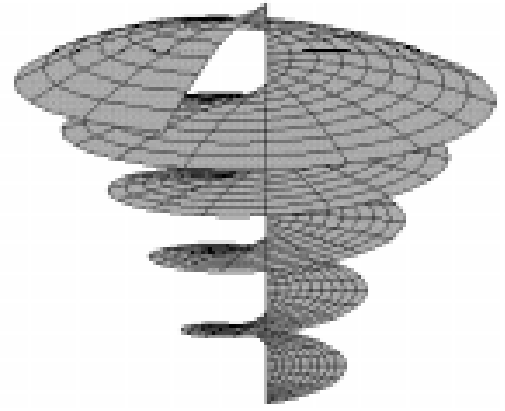

b

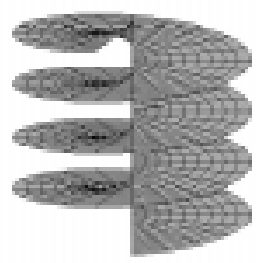

Fig. 2. Wave surface matching of a CV vortex in a void (a) and fiber (b) at the moment of excitation.

Homogeneous and unhomogeneous vortices have different propagation constants $\beta_{1}$ and $\beta_{2}$. Their fields should belong to different topological sets. Inside every set, the fields are transformed into each other with a simultaneous change $l \rightarrow-l, \sigma_{z} \rightarrow-\sigma_{z}$. Inside a set, $\mathrm{CV}$ vortices have similar propagation constants $\beta$.

While exciting a fiber by a circularly polarized light, fields at the input fiber end are matched according to the following boundary conditions:

$\mathbf{e}_{\mathrm{t} 1}=\mathbf{e}_{\mathrm{t} 2}$ and $\quad \mathbf{h}=\mathbf{h}$.

These conditions permit to match smoothly the free space optical vortices and guided fiber vortices. The fields corresponding to conditions (15) will be those of homogeneous and inhomogeneous $\mathrm{CV}$ vortices represented by (10) and (11). However, for optical fields in the free space with $l=+1$ and $\sigma_{z}=-1$ or $l=-1$ and $\sigma_{z}=+1$, this matching is not valid. It is clear that the superposition of fields (12) and (13) satisfies the condition (15) for $z=0$ which schematically could be presented as:

$\mathrm{IV}_{-1}^{+} \Rightarrow \mathrm{TM}+\mathrm{iTE}$

$\mathrm{IV}^{-} \Rightarrow \mathrm{TM}-\mathrm{iTE}$.

For $z=0$, fields (16) and (17) are homogeneously circularly polarized in a cross section, and have a purely screw dislocation of the wave front of the transversal field with $|l|=1$. Since the propagation constant of TE and TM modes is $\beta_{2} \neq \beta_{4}$ then, as it follows from expressions (12), (13), (16), (17), such mode combinations are accompanied with beatings between the fields of partial vortices: $\hat{\mathbf{e}}^{+} F_{1}(R) \exp \{-i \varphi\} \quad$ and $\quad \hat{\mathbf{e}}^{-} F_{1}(R) \exp \{+i \varphi\}$. The superposition of the fields (16) and (17) is called [18, 20] unstable inhomogeneous IV vortices ("IV" means "instability vortex"). The topological charge and helicity $\sigma_{z}$ in an IV vortex are indefinite. However, in some cross sections of the fiber the conversion of states $\left|\sigma_{z}, l>:\right|+1,-1>\leftrightarrow \mid-1$, $+1>$ takes place. IV vortices are not eigenmodes of the optical fiber.

\section{Angular momentum flux of optical vortex fields}

We shall study the ability of an optical fiber to transport the angular momentum when we generate an optical field of the optical vortex type in it. To study whether radiation can transport the angular momentum, one has to use the tensor of the angular momentum flux density [21].

As shown in [11, 12, 22-24], in optical fields with axial symmetry vortices may possess their own angular momentum.

The vector $\mathbf{K}$ of the total angular momentum flux through a surface $\mathrm{S}$ is given by the expression:

$K_{i}=\iint_{S} \Pi_{k i} d S_{k}$,

where $\Pi_{k i}$ is the tensor of the angular momentum flux density:

$\Pi_{k i}=\varepsilon_{i m n} r_{m} T_{n k}$.

Here $\varepsilon_{i m n}$ is an absolutely asymmetric tensor, $T_{n k}$ is the Maxwell tension tensor

$T_{n k}=E_{n} D_{k}+H_{n} B_{k}-\frac{1}{2} \delta_{n k}(\vec{E} \cdot \vec{D}+\vec{H} \cdot \vec{B})$.

If $S$ is an arbitrary cross-section of the fiber, the flux vector is given by the formula:

$K_{i}=\iint_{S} \Pi_{3 i} d S_{3}$

where $d S_{3}=d x d y$. To obtain the time-averaged value of $\mathbf{K}$, one can use the following method of averaging the products of monochromatic fields:

$<A B>=\frac{1}{2} \operatorname{Re} A B *$,

where $<>$ means time-averaging.

The structure of homogeneous vortices may be presented through the expressions (10).

Direct calculations show that $\left\langle\Pi_{32}\right\rangle$ and $\left\langle\Pi_{31}\right\rangle$ are proportional to $\sin \varphi$ and $\cos \varphi$ respectively, therefore after integrating by $\varphi$ their contributions to $\langle\mathbf{K}\rangle$ disappear. For $<\Pi_{33}>$ we have

$<\Pi_{33}>=\mp \rho_{0}^{3} R F_{l}(R) G_{l}^{-}(R) \frac{\sqrt{2 \Delta}}{V} \varepsilon_{0}\left(n_{c o}^{2} \mu+\varepsilon\right)$,

where e and $\mathrm{m}$ are constants of the material on the fiber axis. 


\section{K. N. Alexeyev et al.: Optical vortices and angular momentum...}

To obtain a physically meaningful result, we have to divide the total angular momentum flux through a cross-section $\left\langle\Pi_{33}\right\rangle$ by the total energy flux through the same cross-section $\left\langle P_{3}\right\rangle$. The density of the energy flux is given by the z-component of the Pointing vector $\mathbf{P}=\mathbf{E} \times \mathbf{H}$. In our case, for $\left\langle P_{3}\right\rangle$ we have:

$$
<P_{3}>=2 a_{t}^{2} n_{c o} \sqrt{\frac{\varepsilon_{0}}{\mu_{0}}} F_{l}^{2}(R),
$$

where $a_{t}$ is the magnitude of the transversal electric field.

Since $F_{l}(R)$ rapidly vanishes when $R \rightarrow \infty$ and is finite at $R=0$, we have an evident relation:

$$
\int_{0}^{\infty} R^{2} F_{l}(R) G_{l}^{\mp}(R) d R=-(l \pm 1) \int_{0}^{\infty} R F_{l}^{2}(R) d R .
$$

Using this relation and the fact that for optical fibers $\mu=1$, we finally obtain the result:

$\mathrm{t}=\frac{<K_{3}>}{<N_{3}>} \approx \pm \frac{l+1}{\omega}$

where $\omega$ is the radiation frequency and $<N_{3}>=\iint_{S}<P_{3}>d S_{3}$.

The structure of unhomogeneous vortices may be presented by expressions (11).

Since the result of calculations is similar to those described above, we finally obtain:

$t=\frac{<K_{3}>}{<N_{3}>} \approx \pm \frac{l-1}{\omega}$.

The result is valid for $l>1$.

The structure of IV vortices $(l=1)$ has the form (12), (13), (16), (17).

These vortices consist of two TM and TE modes with different propagation constants $\beta: \beta_{T M}$ and $\beta_{T E}$, respectively. Therefore, the field of the vortices is subjected to a mode dispersion and in this way they are unstable and their angular momenta are zero (see (26)).

From the expressions (14), (18)-(20) it follows that the $\mathrm{HE}_{11}$ mode field transports the angular momentum, and its value is:

$t=\frac{<K_{3}>}{<N_{3}>} \approx \pm \frac{1}{\omega},(\mathrm{k}= \pm 1)$

In the general case, for all kinds of vortices we have

$$
\tau_{l}^{\sigma}=\kappa \frac{l+\sigma}{\omega}
$$

where $k$ is a so-called spin-orbital interaction index [25]. This expression coincides with the expression presented in [11] where the only difference is $k=l \sigma_{z}$ coefficient.

\section{Ray surface of Gaussian beams and $\mathrm{CV}$ vortices}

The field structure of optical vortices in a void is convenient to represent in a form of lines based on the Pointing vector $\mathbf{P}=\mathbf{e} \times \mathbf{h}[12]$. However, the vector $\mathbf{P}$ satisfies the continuity equation:

$\nabla \mathbf{P}+\partial \mathrm{W} / \partial \mathrm{t}=0$

where $\mathrm{W}(\mathrm{x}, \mathrm{y}, \mathrm{z})$ is the energy density.

The energy flow $\mathbf{P}$ is determined up to a vector $\nabla \times \mathbf{A}$.

$A$ vivid presentation of the energy flow $\mathbf{P}$ in an optical vortex and the actual physical picture of the field may be adjusted with each other by means of the Erenfest theorem [26]. If a ray set is characterised by a Pointing vector $P$, then the possibility to find a ray of light at a given point of space, according to the Erenfest theorem, is a module square of the normalised wavefront function $|\psi|^{2} \sim|\mathbf{e}|^{2}$. (In particular, this theorem is strictly observed in a void, in homogeneous and parabolic media). Therefore, from the whole set of vector $\mathrm{P}$ ray lines only those are selected that are located at the distance $r=r_{\max }$ from the axis $\mathrm{z}$ and satisfy the condition $|\mathbf{e}|^{2}=\left|\mathbf{e}_{\max }\right|^{2}$. We call the set of these lines a ray surface.

Let us represent the electric (magnetic) field in the form:

$$
\mathbf{e}(x, y, z)=\mathbf{e}(x, y, z) \exp \left\{i k_{0} \mathfrak{I}(x, y, z)\right\}
$$

where $k_{0}=2 \pi / l_{0}, \mathbf{e}(\mathrm{x}, \mathrm{y}, \mathrm{z})$ è $\mathfrak{I}(\mathrm{x}, \mathrm{y}, \mathrm{z})$ are slowly changing functions. Then, from the Maxwell equation we find

$$
\mathbf{P}=\frac{1}{4} \sqrt{\frac{\varepsilon_{0}}{\mu_{0}}}\left\{\mathfrak{e}^{2} \nabla \mathfrak{I}+\frac{i}{2 k}\left[\left(\mathbf{e} \nabla \mathbf{e}^{*}-\mathbf{e}^{*} \nabla \mathbf{e}\right)+\left(\mathbf{e} \times \nabla \times \mathbf{e}^{*}-\mathbf{e}^{*} \times \nabla \times \mathbf{e}\right)\right]\right\}
$$

The equation $\mathfrak{I}(\mathrm{x}, \mathrm{y}, \mathrm{z})=$ const characterises the shape of the wavefront, therefore $\nabla \mathfrak{I}(\mathrm{x}, \mathrm{y}, \mathrm{z})$ presents a set of normals to the wavefront. If we denote the magnitude of the optical vortex as $\psi$ [4], then the Pointing vector components may be written in the form:

$$
\begin{aligned}
& P_{r}=-\omega \varepsilon_{0}|\psi|^{2} \partial(\arg \psi) / \partial r, \\
& P_{j}=-\omega \varepsilon_{0} / r|\psi|^{2} \partial(\arg \psi) / \partial \varphi+\omega \varepsilon_{0} \sigma \partial|\psi|^{2} / \partial r, \\
& P_{z}=-\omega \varepsilon_{0}|\psi|^{2} \partial(\arg \psi) / \partial z+\omega k \varepsilon_{0}|\psi|^{2} .
\end{aligned}
$$

It is obvious that the magnitude argument function $y$ characterises the wavefront surface: $\arg (\psi)=\mathfrak{I}$ in expression (30). Then, comparing (32) with (31), we find that a deviation of the vector $\mathbf{P}$ from the direction normal to the wavefront is basically determined by the second term in expression (32) for the $\mathrm{P}_{\varphi}$ component. For a linearly polarised light $(\sigma=0)$, the vector $\mathbf{P}$ is perpendicular to the wavefront surface $\mathfrak{I}$. The vector $\mathbf{P}$ experiences the maximum deviation from the normal for a circularly polarised light $(\sigma= \pm 1)$.

Nevertheless, from expression (32) and equation $d \varphi / P_{\varphi}=d z /\left(r P_{z}\right)$ we have

$\varphi=\tan ^{-1} z / z_{R}$ 


\section{K. N. Alexeyev et al.: Optical vortices and angular momentum...}

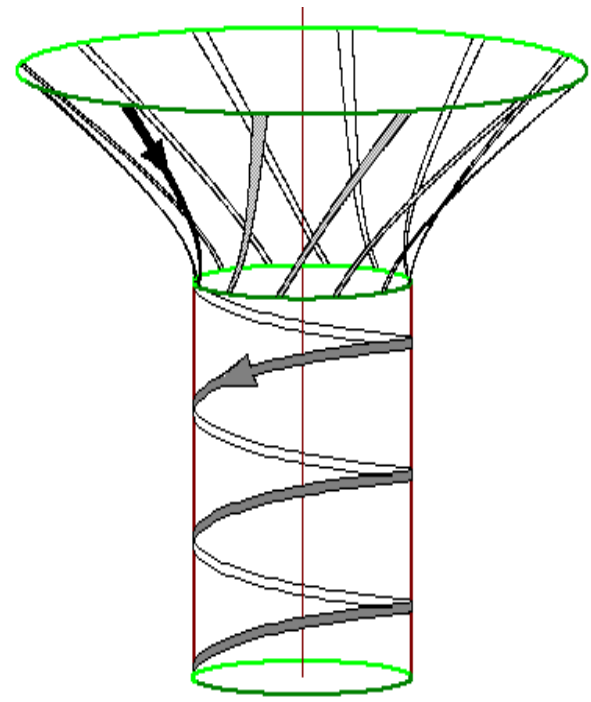

a)

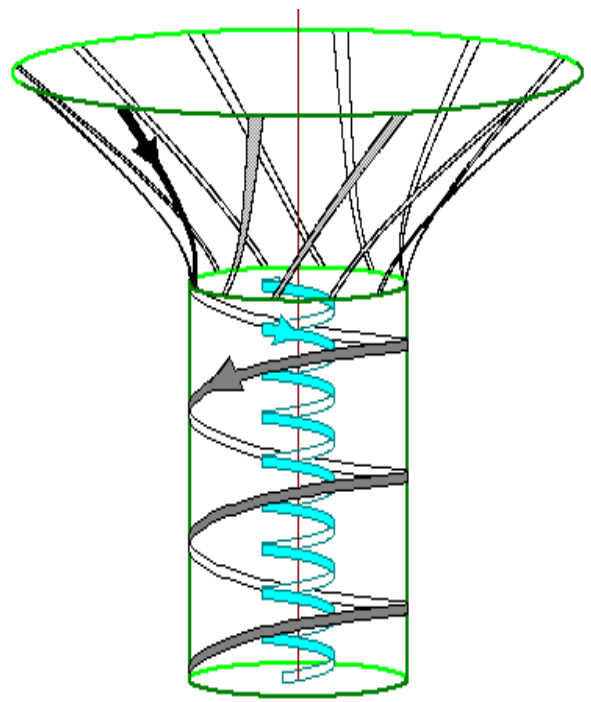

b)

Fig. 3. Ray-surface matching of homogeneous (a) and inhomogeneous (b) CV vortices at the moment of fiber excitation.

( $z_{R}$ is the Rayleigh length). It means that the azimuth evolution of the angle $\varphi$ is determined by Gouy phase and does not depend on the topological charge $l$ and helicity $\sigma$. A similar expression was obtained in the paper [27] for a linearly polarised Gaussian beam.

Fig. 3 shows the ray surface for optical vortices, building up at the radius $r_{\max }^{(b)}=\sqrt{l / 2 w} w(z)(w(z)$ is the halfwidth of the beam), at which the magnitude $\varphi$ is maximum. From this picture, it is seen that the generatrices of the ray surface are straight rays which do not cross axis $z$. To construct the ray surfaces of homogeneous and inhomogeneous CV vortices in a multimode fiber, the Pointing vector components are found from expressions (10) and (11) in the following form:

$P_{r}=0 ; P_{\varphi}=K G_{l}^{-k} F(R) ; \quad P_{z}=K \frac{V}{\sqrt{2 \Delta}} F_{l}(R)$,

where $k=+1$ for a homogeneous and $k=-1$ for an inhomo-

geneous vortex, $K=a_{1}^{2} /\left(2 c^{2}\right) n_{c o}\left(\varepsilon_{0} / \mu_{0}\right)^{1 / 2}(\sqrt{2 \Delta} / V)$, $a_{1}$ is the electric field amplitude.

The ray surface of a homogeneous CV vortex is presented by fig. 3(a) where $l \cdot \sigma>0$. The ray surface presents a set of helices wound on a circular cylinder. The radius of this cylinder $r_{\max }^{(f)}$ is equal to the radius of the Gaussian beam $r_{\max }^{(b)}$.

An inhomogeneous $\mathrm{CV}$ vortex, for which values 1 and s have opposite signs, has a more complicated shape of the ray surface (fig. 3(b)). The ray surface splits into two cylinders. The generatrix helices of these cylinders have oppo- site signs of twisting. For an essentially multimodal fiber $(V \rightarrow \propto)$, the radius of the inner cylinder $R_{i n} \rightarrow 0$, the ray surface is degenerated into a single cylinder with helical raylines. For an unstable IV vortex, the ray surface is degenerated into a cylinder with straight ray lines. It follows that the angular momentum of an IV vortex is equal to zero $\left(\mathrm{M}_{\mathrm{IV}}=\right.$ $=0$ ).

This behaviour of the ray surface of inhomogeneous CV vortices is associated with the contribution of the spin and orbital angular momentum to the total angular momentum: the difference of signs for $l$ and $s$ results in a decrease of the total angular momentum.

The screw character of the generatrix lines of a CV vortex ray surface is caused by the effect of the refractive index inhomogeneity $\tilde{\mathrm{N}}\left(\ln n^{2}\right)$ curving the energy flow lines of vector $\mathbf{P}$.

Matching the Gaussian beam ray surfaces and a CV vortex at the moment of fiber excitation assumes the equality of the radii of the Gaussian beam ray surface at $z=0$ and the $\mathrm{CV}$ vortex ray surface $\left(r_{\max }^{(f)}=r_{\max }^{(b)}\right)$.

In general, the process of the propagation of a CV vortex along an optical fiber may be schematically represented in the form of two energy flows (fig. 4).

The first circularly polarised light flow $P_{z}$ is directed along the fiber axis $z$. The second linearly polarised flow $P_{\varphi}$ circulates in the direction $\hat{\mathbf{e}}_{\varphi}$, which is orthogonal to the fiber axis.

The presence of the azimuth flow $P_{\varphi}$ is associated with the appearance of a «surface» wave which propagates perpendicularly to the direction of the main flow (see, for example [28-30]). 


\section{K. N. Alexeyev et al.: Optical vortices and angular momentum...}

\section{Continuity equation}

There are two problems in the study of the angular momentum of radiation. The first one is concerned with a question whether radiation itself may accumulate an angular momentum. To answer it, one has to integrate the density of the orbital angular momentum given by the expression (19) over the volume $\Omega$. For monochromatic fields in axially symmetrical fibers, the time-averaged density $\mathbf{M}$ does not depend on $z$ and the averaged density of energy. Therefore

$$
\frac{\mathbf{L}}{E}=\frac{\int{ }_{\Omega} \mathbf{M} d \Omega}{E}=\frac{\iint_{S} \mathbf{M} d S}{E / a},
$$

where $\mathbf{L}$ is the total angular momentum of radiation, $E$ is the radiation energy, $\alpha$ is the distance between two crosssections bounding the volume $\Omega$. The nominator in (35) has the meaning of the linear density of energy, while the numerator acquires the form of a flux. However, though it is the flux of the vector of the angular momentum density, it is not the flux of the angular momentum in an arbitrary situation. This delusive similarity may lead to some mistakes since the true angular momentum flux is given by the expression (18). Moreover, for situations when $|\mathbf{M}|$ depends on $z$, the last equality in (35) does not take place, and the flux $\iint_{S} \mathbf{M} d S$ has no physical sense.

The second problem arises when we study the ability of radiation to transport the angular momentum. In this case, one has to use the formalism based on the continuity equation and related concepts. This is the problem we study in this paper. Let us demonstrate that our main result is in good agreement with some predictions of the general theory.
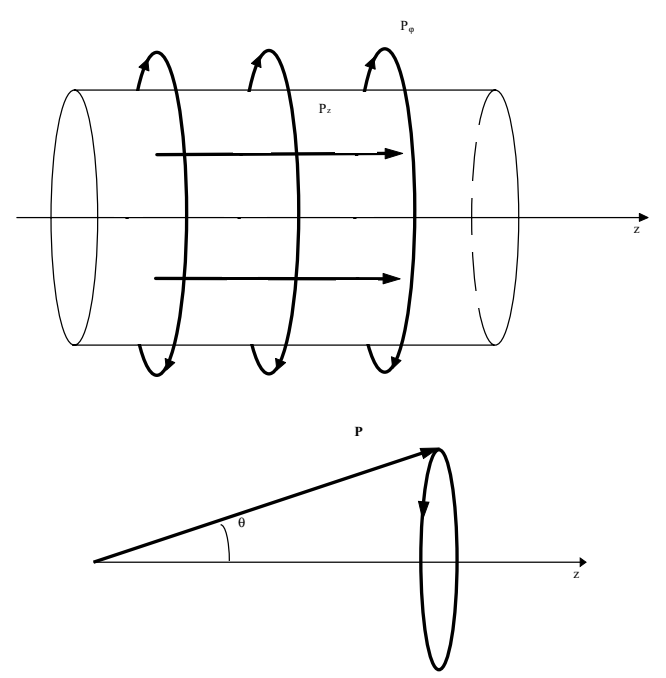

Fig. 4. Axial $P_{z}$ and azimuthal $P_{\varphi}$ energy flows of a CV vortex (a) and Pointing vector precession (b).
The continuity equation for the angular momentum in inhomogeneous media has the following form:

$$
\frac{\partial M_{i}}{\partial t}+\frac{\partial \Pi_{i k}}{\partial x_{k}}=\frac{1}{2}(\mathbf{r} \times \nabla \varepsilon)_{i} E^{2}
$$

where $\varepsilon$ is the dielectric permeability, $i, k=1,2,3$.

For monochromatic fields $\left\langle M_{i}\right\rangle$ does not depend on t, and by averaging (36) we obtain:

$$
\frac{\partial}{\partial x_{k}}\left\langle\Pi_{i k}\right\rangle=\frac{1}{2}(\mathbf{r} \times \nabla \varepsilon)_{i}\left\langle E^{2}\right\rangle .
$$

For systems with axial symmetry and a rapid decrease of fields to the infinity from the axis it is equivalent to

$\iint_{S_{3}}\left\langle\Pi_{3 з}\right\rangle d S_{3}=$ const,

where $S_{3}$ is an arbitrary cross-section. It means that the flux of the angular momentum through a cross-section does not depend on the $z$-coordinate of the cross-section. Thus, the results (22), (24) and (26) are to be regarded as consequences of the angular momentum conservation law in its differential form (36).

In an arbitrary situation, there is an essential difference between these two aspects of the problem of the angular momentum of a field. For instance, a constant electric field possesses no angular momentum, but still has a non-zero density of the angular momentum flux. However, in propagating electromagnetic fields these differences are smoothened to a certain extent. It turns out that for nonmagnetic weak-guided fibers $(\mu=1)$ the ratio $\langle L\rangle /\langle E\rangle$ estimated with the help of (35) equals the ratio $\left\langle K_{3}\right\rangle /\left\langle N_{3}\right\rangle$ for all $\mathrm{CV}$ vortices.

The right side of (36) presents the change of the angular momentum due to deformations of fiber's cross-section or an anisotropy of the material caused by pseudosinks and pseudosources of the angular momentum.

\section{Conclussion}

A form of existence of circularly polarized eigen modes of optical fibers is guided optical vortices. The field of a fundamental $\mathrm{HE}_{11}$ mode $(l=0)$ has only a purely screw dislocation of the wavefront of the longitudinal component. All fields with azimuth index $l \neq 0$ form two sets of eigen modes: homogeneous $\mathrm{CV}$ vortices, inhomogeneous $\mathrm{CV}$ vortices $(l \neq 0,1)$, and $\mathrm{TE}_{0 \mathrm{~m}}$ and $\mathrm{TM}_{0 \mathrm{~m}}$ modes consisting of a superposition of partial IV vortices. For optical fiber vortices, a selection rule is satisfied. If the sum of a topological charge and polarisation $l+\sigma_{z} \neq 0$, then the vortex in an optical fiber is structurally stable (CV vortices). If $l+\sigma_{z}=0$, then the vortex is structurally unstable (IV vortices). 


\section{K. N. Alexeyev et al.: Optical vortices and angular momentum...}

Fields of stable optical vortices are able to transmit angular momentum. The relation of the angular momentum flow in $z$ direction to the $z$ component of the Pointing vector of a multimode fiber field is equal to $k\left(l+\sigma_{z}\right) / w$. This expression is very similar to that for optical vortices in a free space.

Fields of unstable IV vortices, TE and TM modes do not transmit any angular momentum.

The field of optical vortices is characterised by two kinds of surfaces: the wavefront surface of transversal components of the electric vector $\mathbf{e}_{\mathbf{t}}$ has the shape of a straight helicoid both for optical vortices in a void and $\mathrm{CV}$ vortices in a multimode fiber. The distance between the neighbouring leaves of the wavefront helicoid is equal to the wavelength $\lambda=v / v$, where $v$ is the phase velocity of the vortex.

The ray surface is formed by Pointing vector $\mathbf{P}$ lines situated at the distance $r_{\max }$ from the energy flow maximum. The ray surface of optical vortices in a void for $m=1(\mathrm{~m}$ is radial index) has a form of a rotation hyperboloid formed by straight lines. The ray surface of $\mathrm{CV}$ vortices presents a helix set wound around a circular cylinder.

Upon fiber excitation, matching of an optical vortex in a void with a guided $\mathrm{CV}$ vortex assumes matching the wave and ray surfaces. The exact matching of wave surfaces means that the vortex in a void excites only the given $\mathrm{CV}$ vortex in the fiber.

In case of mismatch of the wave surfaces, other fiber eigenmodes could be excited. The matching degree of the ray surfaces indicates the share of energy that was transferred from a Gaussian beam to a guided CV vortex.

The conservation law for the angular momentum in a fiber is given by the continuity equation. For an axially-symmetrical fiber cross-section the right side of the continuity equation is equal to zero. However, if the cross section of the fiber is changed due to a deformation, or there is an anisotropy of the material, then the right side of (28) describes pseudosinks or pseudosources of the angular momentum.

\section{References}

1. J. F. Nye, M. V. Berry, Proc. R. Soc. Lond. A, 336, 165 (1974).

2. M. Harris, C.A. Hill, J. M. Vaughan, Opt. Comm., 106, 161, (1994)
3. V. Yu. Bazhenov, M. S. Soskin, M.V. Vasnetsov, Journ. of modern optics, 39, No.5, 985 (1992).

4. I. V. Basistiy, V. Yu. Bazhenov, M. S. Soskin, M. V. Vasnetsov, Opt. Comm., 103, 422 (1993).

5. E. Abramochkin, V. Volostnikov, Opt. Comm., 83, 12, 123 (1991).

6. B. Ya. Zel'dovich, N. F. Pilipetskiy, V. V. Shkunov, Principles of phase conjugation, Springer Series Optics Science, 42, Berlin, (1985).

7. A. V. Volyar, S. N. Lapayeva, T. A. Fadeyeva, Techn. Phys. Lett., 20, No.3, 187 (1994)

8. A. V. Volyar, T. A. Fadeyeva, Lett. to Journ. Techn. Phys., 23, No.2, 20 (1997). (in Russian).

9. A. V. Volyar, T. A. Fadeyeva, Techn. Phys. Lett., 22, No.4, 330 (1996)

10. V. Yu. Bazhenov, M. S. Soskin, M. V. Vasnetsov, Experimental and Theorethical Physics Journal Letters, 52, No.8, 1037 (1990).

11. M. Ya. Darsht, B. Ya. Zel'dovich, I. V. Katayevskaya, N. D. Kundikova, Journ. of Theor. and Experim. Phys. 1995. V.107. No.5. P.1464-1472. (in Russian).

12. L. Allen, M. W. Beijersbergen, R. J. Spreeuw, J. P. Woerdman, Phys. Rev. A., 45, No.11, 8185 (1992).

13. M. E. J. Friese, H. He, N. R. Heckenberg, H. Rubinsztein-Dunlop, Proc. SPIE, 2792, 190 (1995).

14. S. M. Barnett, L. Allen, Opt. Comm., 110, 670, (1994).

15. H. He, M. E. Friese, N. R. Heckenberg, H. Rubinsztein-Dunlop, Phys. Rev. Lett., 75, No.5, 826 (1995)

16. A.W. Snyder, J. D. Love, Optical Waveguide Theory, Chapman and Hall, London, (1983)

17. A. V. Volyar, T. A. Fadeyeva, Singular optics of low-mode fiber fields: I. The circular disclinations, Optics and Spectroscopy, (1998) (to be published), (in Russian).

18. A. V. Volyar, T. A. Fadeyeva, Techn. Phys. Lett., 22, No.4, 333 (1996)

19. J. F. Nye, Proc. R. Soc. Lond A., 387, 105 (1983).

20. A. V. Volyar, T. A. Fadeyeva, H. M. Reshitova, Lett. to Journ. Techn. Phys., 23, No. 5, 70 (1997) (in Russian).

21. L. D. Landau, E. M. Lifshits, Theoretical physics V.2 Theory of field, Nauka, Moskow, 509, (1988). (in Russian).

22. A. V. Volyar, T. A. Fadeyeva, Lett. to Journ. Techn. Phys., 23, No.21, 74 (1997) (in Russian).

23. A. V. Volyar, T. A. Fadeyeva, Lett. to Journ. Techn. Phys., 23, No.22, 58 (1997) (in Russian)

24. A. V. Volyar, T. A. Fadeyeva, Lett. to Journ. Techn. Phys., 23, No.23, 59 (1997) (in Russian).

25. A. V. Volyar, T. A. Fadeyeva, V. G. Shvedov, V. Z. Zhilaytis, Opt. and Spectr. (1998) (to be printed) (in Russian)

26. D. Marcuse, Light Transmission Optics, van Nostrand Reinhold Company, New York, 570, (1972).

27. M. J. Padgett . L. Allen, Opt. Comm., 121, 36 (1995).

28. C. Imbert, Phys Rev.D, 5, No.4, 767 (1972).

29. A. V. Volyar, Yu. N. Mitzay, S. N. Lapayeva, Techn. Phys. Lett., 20, No.3, 190 (1994)

30. A. V. Dooghin, N. D. Kundikova, V. S. Liberman, B. Ya. Zel'dovich, Phys. Rev. A., 45, No.11, 8204 (1992).

\section{ОПТИЧНІ ВИХОРІ ТА ПОТІК ЇХНЬОГО КУТОВОГО МОМЕНТА В БАГАТОМОДОВИХ ВОЛОКНАХ}

О. М. Алексієв, Т. А. Фадєєва, О. В. Воляр, М. С. Соскін

Фізичний факультет Сімферопольского Державного Університету, Украӥна

"Інститут фізики НАН Украӥни

Резюме. Розглядається проблема розповсюдження оптичних вихорів в багатомодових волокнах. Показано які структирнихзмін дізнають хвильова і променева поверхні при переході із вільного простору в середовище волокна. Записане рівняння неперервності для потоку кутового момента вихоря в неоднорідному середовищі.

\section{ОПТИЧЕСКИЕ ВИХРИ И ПОТОК ИХ УГЛОВОГО МОМЕНТА В МНОГОМОДОВЫХ ВОЛОКНАХ}

\section{О. М. Алексеев, Т. А. Фадеева, О. В. Воляр, М. С. Соскин \\ Физический факультет Симферопольского Государственного Университета, Украина \\ "Институт физики НАН Украины}

Резюме. Рассматривается проблема распространения оптических вихрей в многомодовых волокнах. Показано какие структурные изменения испытывают волновая и лучевая поверхности при переходе из свободного пространства в среду волокна. Записано уравнение непрерывности для потока углового момента вихря в неоднородной среде. 